\title{
Optimal Routing and Scheduling in Multihop Wireless Renewable Energy Networks
}

\author{
MHR. Khouzani*, Saswati Sarkar*, Koushik $\operatorname{Kar}^{\dagger}$ \\ * University of Pennsylvania \\ $\dagger$ Rensselaer Polytechnic Institute \\ Philadelphia, PA 19104, USA \\ Troy, NY 12180, USA \\ Email: \{swati,khouzani\}@ seas.upenn.edu \\ Email: kark@rpi.edu
}

\begin{abstract}
In this paper, we design routing and scheduling policies that optimize network throughput in energy-constrained wireless ad-hoc networks where nodes are powered by renewable energy sources. We take into account the fact that renewable energy harvesting processes are unpredictable and stochastic in nature - typically depending on environmental factors that are not known in advance. The routing and scheduling policies that we propose do not require explicit knowledge of the statistics of the energy harvesting or the traffic generation processes, and are able to dynamically learn and adapt to time variations in the physical and network environments, so as to deliver data rates that are optimal in the long term. We obtain bounds on the capacity of the energy storage devices at the individual nodes that is minimally required for obtaining maximum throughput in the network; we also compute what fraction of the throughput region is attained when the energy storage capacity is less than this limit.
\end{abstract}

\section{INTRODUCTION AND BACKGROUND}

In recent years, there has been a significant amount of interest - both in the academia and the industry - in developing wireless systems and networks that are powered by renewable energy sources like solar, wind, vibration etc. In fact, multiple vendors, e.g. Meraki [1], Proxim Wireless [2], currently provide wireless mesh nodes that can be attached to solar panels. In addition to solar, energy harvested from the wind through the use of micro-turbines [3], or from vibration of infrastructure (like bridges and tall poles) [4] could potentially be used for powering wireless nodes in the near future. Use of renewable energy has advantages in terms of operation cost and environment-friendliness, and in many cases may be the only option available due to practical constraints. For wireless adhoc or sensor networks deployed in an area that does not have any existing infrastructure, reliance on renewable energy sources become necessary. Examples include sensor networks deployed in inaccessible environments for data collection. Even if the wireless networking devices have continuous or intermittent access to the power line, it is may be desirable - to minimize cost and environmental hazards - to make the best use of the renewable energy, and and draw energy from non-renewable sources only when the amount of available renewable energy is not enough. Indeed, it could be argued that the true power of "wireless" networks could be realized only when the wireless nodes would not require wired connectivity to the power line, or rely on batteries which will eventually run out. Not surprisingly, therefore, there has been several recent studies on different aspects of network design, operation and optimization when the network nodes gather power from renewable energy sources in the environment [5]-[10], particularly in the context of sensor networks.

Powering networks using renewable energy comes with significant design and optimization challenges. Firstly, the rate of energy available from renewable sources is typically small, implying that energy can become the major constraint in all network operations. The rate of energy harvested from renewable sources could range from the order of tens of watts as with solar panels, to milli or micro watts as with micro-turbines or vibration sensors. Indeed, the choice of the renewable energy source would need to be determined based on the application scenario, along with other practical constraints. For example, a solar panel (with several square feet of panel area) may be more appropriate for a WiFi access point which consumes energy in watts to tens of watts. On the other hand, a set of micro-turbines may be adequate for a small sensor (like $2.4 \mathrm{GHz}$ ZigBee mote) meant for infrequent sensing and transmission of information, and may be desirable from the perspectives of concealment and ease of deployability (often important in sensor network applications). In these scenarios, the energy harvesting rates will typically be of the same order, just enough or even barely necessary, to maintain the essential networking functions. Therefore, energy usage must be carefully optimized in all network operations. Secondly, the energy replenishment process is highly variable, and is governed by random environmental factors that can be neither controlled nor accurately predicted. For example, while solar insolation is closely tied to the weather, predicting how it will evolve at the exact wireless node location is in general difficult. Wind speeds at specific locations are even more unpredictable, both at hourly and daily time-scales. Vibration energy may depend on unpredictable factors like number of vehicles going on/past a bridge or a pole, as well as wind speeds. In addition, the energy replenishment process at a single wireless node, or across different nodes in the network, are likely to show a significant degree of temporal and spatial correlations, which are again difficult to estimate in advance. These factors imply that to ensure that the network remains operational at all times, energy-intensive network functions must be optimized taking into account stochastic variations in the energy renewal process at the different node locations. This in general involves a combination of statistical 
estimation or prediction of the energy replenishment process, and stochastic optimization on network functions based on such estimation/prediction. Thirdly, wireless nodes may be subject to limitations in their energy storage capabilities, due to limitations in the battery size for example. This is particularly true for sensor networks, where the sensor devices (and hence their batteries) may be constrained to be small in size. Energy storage limitations impose additional complications on optimal energy usage policies - intuitively, nodes with larger amount of energy (or nodes whose energy storage unit is closer to being full) at any time should be preferentially used for energyintensive network functions.

In this paper, we consider the questions of routing and scheduling in general multi-hop wireless networks where nodes are powered through random time-varying renewable energy processes. We design joint routing and scheduling algorithms that are stochastically optimal, in the sense that they maximize end-to-end data throughput in the network. In the algorithms we propose, the energy renewal process estimation/prediction and the network function optimization components are integrated together; thus our algorithms do not need to know the energy replenishment rates, and can dynamically learn and adapt to their variations. The solutions and optimality results also admit a very general correlation structure among the energy replenishment processes at the different nodes in the network, which need not be known in advance. Our algorithms do not require a priori knowledge of the data traffic generation rates either, and achieve the maximum throughput region of the network that takes into account both energy and and interference constraints in the wireless network. In our analysis, both transmission and reception energy costs are taken into consideration, and the effect of limited energy storage at nodes is also explored.

More specifically, the novel contributions of this work are as follows. We obtain integrated routing and transmission scheduling policies that attain the maximum throughput region in a renewable energy powered wireless multi-hop network. We argue that energy storage limitations can affect the maximum network throughout attainable, and obtain bounds on the capacity of the energy storage devices at the individual nodes that is minimally required to attain maximum throughput. Alternatively, our results can be used to compute what fraction of the maximum throughput region can be attained when the energy storage capacity is less than this limit. Our framework and results are applicable to both wireless ad-hoc and sensor networks, which we do not distinguish explicitly in our model.

The analysis techniques we use draw from those used in [18], [20]-[22] for throughput-optimal routing and scheduling in non energy-constrained networks. However, as we explain later in the paper, these results do not directly extend to renewable energy networks, due to the fact that unlike channel availability, energy can be stored, thereby introducing dependencies between the evolution of the packet and the energy queues. We overcome this challenge by using two or more (depending on whether reception energy is negligible or not) packet queues per node/link (instead of using only one), and a novel energy marking based packet forwarding control policy. The additional complexity in the routing and scheduling policy introduced due to renewable energy considerations requires message exchanges that are local in nature - a nice feature of our algorithm. Interestingly, we also show that the energy storage requirements for attaining any desired fraction of the network stability region can be upper bounded by a constant, quite unlike similar requirements on packet buffering where the bound grows with the network size.

While the question that we formulate and address in this paper is novel, there are some related recent work in algorithm design and performance optimization of renewable energy networks that are worth mentioning and contrasting with. The problem of adaptive sleep scheduling (node activation) in renewable energy (rechargeable) sensor networks have been considered in [11]-[13], which involves determining when each node should be put to sleep to conserve energy for optimal long-term performance. In contrast, we assume that nodes are always powered on, but focus on communication issues like packet routing and scheduling to optimize energy usage. [14] develops transmission power and packet admission control policies in this context, towards maximizing some suitably defined long-term user satisfaction measure. [16] considers the question of determining the sampling rates and routes in a renewable energy sensor network. This work does not take scheduling into account, and presents a deterministic sub-gradient based algorithm for this purpose. In contrast, we cast and prove the optimality of our joint scheduling and routing policy in a stochastic optimization framework. The online routing question in renewable energy networks has been considered in [15] from a competitive analysis perspective; the authors obtain a competitive ratio that is logarithmic in the network size. In our work, we consider both routing and scheduling, and obtain policies that attain the maximum achievable throughput using stochastic optimization techniques. Also, unlike these previous works, we consider the effect of small energy storage (typical of a large class of sensor networks) on the optimality/performance results.

The paper is structured as follows. Section II describes the system model. In Section III, we develop joint routing and transmission scheduling policies that attain throughputoptimality when both energy and capacity/interference constraints are taken into account. In Section V we consider the effect of energy storage capacity limitations on the maximum throughput attained in the network.

\section{System Model And Problem Formulation}

We consider a multi-hop wireless network modeled as a directed graph $G=(V, E)$, where $V$ and $E$ respectively denote the sets of nodes and links, and $|V|=N,|E|=L$. A link exists from a node $u$ to another node $v$ if and only if $v$ can receive $u$ 's signals. The link set $E$ depends on the transmission power levels of nodes and the propagation conditions in different directions. Let $I_{v}, O_{v}$ be the sets of links ending at and originating from any node $v$, respectively. The network has $M$ sessions, or end-to-end flows, $1, \ldots, M$. 
Each session refers to a source(s)-destination(s) pair. Let $\mathcal{M}$ be the set of all the sessions in the network.

We assume that time is slotted. We now describe the packet arrival process for the sessions. Let $A_{m}(t)$ be the number of packets that session $m$ generates at its source node in interval $(t, t+1], m=1, \ldots, M$. We assume that the arrival process $\left\{A_{1}(),. \ldots, A_{M}().\right\}$ is stationary, ergodic with $\mathbf{E}\left(A_{m}(t)\right)=$ $\lambda_{m}$, where $\lambda_{m}$ is referred to as the packet arrival rate of session $m$, and $A_{m}(t) \leq \gamma$ for each $m, t$ for a constant $\gamma$. The packets from different sessions are queues separately at each node. The traffic generated for session $m$ can be routed to its destination (or sink) node via any route in the network, and different packets may follow different routes depending on network congestion and energy availabilities at different nodes which also vary with time.

Next we describe the energy generation, storage and usage processes at each node. Let $E_{i}(t)$ be the number of energy units that node $i$ generates in interval $(t, t+1], i=1, \ldots, N$. We assume that the energy arrival process $\left\{E_{1}(),. \ldots, E_{N}().\right\}$ is stationary, ergodic with $\mathbf{E}\left(E_{i}(t)\right)=e_{i}$, where $e_{i}$ is referred to as the energy arrival rate of node $i$. The energy arrivals at different nodes may be correlated. Node $i$ can store at most $B_{i}$ units of energy, and generated energy is lost if the storage is full. Node $i$ consumes $t_{i}\left(r_{i}\right.$, resp.) units of energy when it transmits (receives) a packet. Let $C_{i}(t)$ be the number of energy units used up by $i$ in interval $(t, t+1], i=1, \ldots, N$, and $P_{i}(t)$ be the number of energy units available at node $i$ at time $t$. Thus, the energy queue, $P_{i}(t)$ evolves as:

$$
P_{i}(t+1)=\min \left(B_{i}, P_{i}(t)+E_{i}(t)-C_{i}(t)\right) .
$$

Definition 1: The packet arrival rate vector $\vec{\lambda}$ and energy arrival rate vector $\vec{e}$ are $M$ - and $N$-dimensional vectors of the packet and energy arrival rates.

We now describe the packet transmission process, which depends on the link scheduling. When a link is scheduled for transmission, it transmits a packet, and energy is consumed at its origin and end nodes. Depending on the wireless interference conditions, certain sets of links can not be scheduled simultaneously. For instance, links $\left(u_{1}, v_{1}\right)$ and $\left(u_{2}, v_{2}\right)$ can not be simultaneously scheduled if $v_{1}$ and $u_{2}$ are close, or $v_{2}$ and $u_{1}$ are close, as it would cause interference at receiver $v_{1}$ in the former case, and at $v_{2}$ in the latter case.

Definition 2: A schedulable set of links is a subset of its links such that all links in the subset can be scheduled simultaneously. Let $J_{1}, \ldots, J_{K}$ be the schedulable sets and let $\vec{J}^{i}$ be the $L$-dimensional indicator vector representing any schedulable set $J_{i}$. Let $\mathcal{J}=\left\{J_{1}, \ldots, J_{K}\right\}$. Any subset of a schedulable set is also a schedulable set.

Definition 3: A routing and scheduling policy is an algorithm that decides in each slot the subset of links that would transmit packets in the slot and the sessions these packets belong to. Clearly, a scheduling policy must designate sessions at each node and subsequently, select an element of $\mathcal{J}$ in each slot, and this element must be such that all links in it have packets to transmit and the sources and sinks of the links in it have the requisite amount of energy for packet transmission and reception.

Every packet has a transmission time of one slot. Thus, if a backlogged link is scheduled in a slot, it transmits a packet in the slot. We assume that any packet arriving in a slot may be transmitted in the next slot.

Let $D_{i}^{m}(t)$ be the number of packets that link $i$ transmits from session $m$ in interval $(t, t+1], i=1, \ldots, N$. Clearly the transmissions depend on the scheduling policy. Let $Q_{m u}(t)$ be the number of packets of session $m$ that are waiting for transmission in node $u$ at the beginning of slot $t$. We assume each packet queue has infinite storage. Note that the arrivals at a node happen due to exogenous packet generation, and also because of transmission on input links to $i$. Thus,

$$
Q_{m i}(t+1)=Q_{m i}(t)+A_{i}^{m}(t)+\sum_{i \in I_{u}} D_{i}^{m}(t)-\sum_{i \in O_{u}} D_{i}^{m}(t) .
$$

Definition 4: The network is said to be stable if $\lim _{T \rightarrow \infty} \sum_{m=1}^{M} \sum_{i=1}^{N} \sum_{t=1}^{T} \mathbf{E}\left(Q_{m i}(t)\right) / T$ is finite.

Definition 5: The stability region of a scheduling policy is the set of packet and energy arrival rate vectors for which the network is stable when the policy is used. A pair of packet and energy arrival rate vectors $(\vec{\lambda}, \vec{e})$ is said to be feasible if it is in the stability region of some scheduling policy. The network stability region is the set of all feasible pair of packet and energy arrival rate vectors.

Our goal is to obtain a routing and scheduling policy that stabilizes the network for any feasible pair of packet and energy arrival rates. In a seminal work, Tassiulas and Ephremides [18], have provided such a policy, that does not require any knowledge of arrival rates, when nodes have unlimited energy reserves. In this paper, we consider the scenario where nodes not only have limited but also randomly varying energy reserves which change (both increase and decrease) with time. Given that energy reserves are limited, an interesting question is whether availability of energy at a node can be treated as availability of links, or rather availability of nodes. As shown in [19], generalizations of the basic policy in [18] stabilizes the network for any feasible pair of packet arrival and link availability rates, even in presence of random fluctuations in link availabilities that may arise due to random fading, node mobility etc. But these results do not apply in our context since energy can be stored (though in limited quantity) unlike channel availability; specifically, if an energy unit is generated in a slot and not used immediately, it may be possible to use it later, but if a link (or node) is up in a slot and no packet is transmitted in it, the transmission opportunity is irretrievably lost. As the following example illustrates, such storage potential can substantially augment the stability region.

Example 1: Consider the simple network with 2 nodes $u, v$ both of which transmit packets to the same destination. All nodes are within each other's transmission range, and hence either $u$ or $v$, but not both, can transmit packets in a slot. Consider correlated energy generation patterns where both $u, v$ generate energy in odd slots and neither generates energy in an 
even slot. The common destination generates energy in each slot. If we consider energy availability like channel availability, i.e., the generated energy must be used immediately after generation and can not be stored for use in other slots, then the nodes can transmit packets only in the odd slots. Thus, since at most one node can transmit in a slot, an arrival rate vector is feasible only if the sum of the arrival rates at the nodes is less than or equal to $1 / 2$. Now, assume that $v$ can store 1 unit of energy, and consider a schedule that allows node $u$ to transmit in odd slots and $v$ to transmit in even slots. Any arrival rate vector that has an arrival rate of at most $1 / 2$ for each node is feasible. Thus, even limited storage increases the stability region increases significantly (is doubled, intuitively).

Owing to the storage potential for energy, the system dynamics is governed by the evolution the packet as well as the energy queues, and the two evolutions are inter-dependent as packet transmissions and receptions deplete the energy queues and modify the packet queues. The challenge now is to determine whether and how such a dependence will affect the policy design. Note that the stability criterion however explicitly depends only on the sizes of the packet queues.

Towards the above goal, we characterize the feasibility conditions for arrival rate pairs. Clearly, the necessary conditions for a pair $(\vec{\lambda}, \vec{e})$ to be feasible is that there exist fractions of time $\omega_{J}^{m}$ associated with the independent sets $J$ and session $m$ such that:

$$
\begin{gathered}
\sum_{J \in \mathcal{J}} \omega_{J}^{m}\left(\left|J \cap O_{v}\right|-\left|J \cap I_{v}\right|\right)=\lambda_{v}^{m}, \underset{k \in \mathcal{M}}{\forall v \in V}, \\
\sum_{\substack{J \in \mathcal{J} \\
m \in \mathcal{M}}} \omega_{J}^{m}\left(t_{v}\left|J \cap O_{v}\right|+r_{v}\left|J \cap I_{v}\right|\right) \leq e_{v}, \forall v \in V, \\
\sum_{\substack{J \in \mathcal{J} \\
m \in \mathcal{M}}} \omega_{J}^{m} \leq 1 ; \quad \omega_{J}^{m} \geq 0, \underset{\substack{J \in \mathcal{J} \\
m \in \mathcal{M}}}{ },
\end{gathered}
$$

We explain each condition. The two conditions in (4) ensure that $\left\{\omega_{J}^{m}\right\}$ are fractions of time. In the first condition, $\left|J \cap I_{v}\right|$ $\left(\left|J \cap O_{v}\right|\right)$ are the number of packets that enter $v$ (leave $v$, resp.) from (to, resp.) other nodes when the schedulable set $J$ is scheduled. Thus, $\sum_{J \in \mathcal{J}} \omega_{J}^{m}\left(\left|J \cap O_{v}\right|-\left|J \cap I_{v}\right|\right)$ is the difference between the output and input rates of packet transmissions for session $m$ at node $v$ (the input rate excludes extraneous packet arrivals and considers only the inputs due to transmission from other nodes). This must equal the sum of the extraneous packet arrival rates of that session due to flow balance. The left hand side of the second constraint is the energy consumption rate at node $v$ which must be upper bounded by the energy generation rate $e_{v} . t_{v}$ and $r_{v}$ respectively denote the per-packet transmission and reception energy consumption at node $v$.

We will show that for large energy storage capacities the above conditions also become sufficient as well, when the equality and inequality in the first two conditions are replaced by strict inequalities. Thus, we will consider packet and energy arrival rate pairs that satisfy:

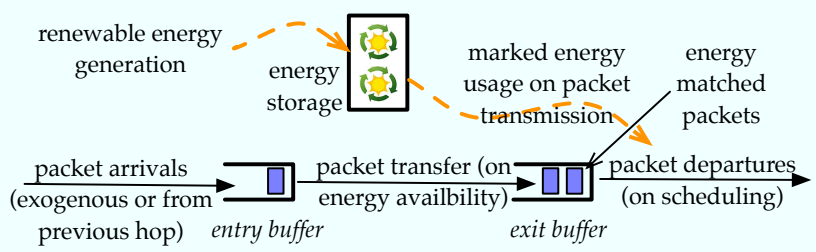

Fig. 1. Packet buffering and energy marking at each node (for negligible reception energy cost, i.e., $r_{v}=0$ ). If $r_{v}>0$, an intermediate buffer and per-link exit buffers are needed (Section IV-B).

$$
\sum_{J \in \mathcal{J}} \omega_{J}^{m}\left(\left|J \cap O_{v}\right|-\left|J \cap I_{v}\right|\right) \geq \lambda_{v}^{m}+\delta, \underset{\substack{\forall \in \mathcal{M} \\ m \in \mathcal{M}}}{\underset{m}{m}}
$$

$$
\sum_{\substack{J \in \mathcal{J} \\ m \in \mathcal{M}}} \omega_{J}^{m}\left(t_{v}\left|J \cap O_{v}\right|+r_{v}\left|J \cap I_{v}\right|\right) \leq e_{v}-\varepsilon, \quad \forall v \in V
$$

for some positive $\delta$ and $\varepsilon$ fractions $\left\{\omega_{J}^{m}\right\}$ that satisfy (4). Let $\Lambda_{\delta, \varepsilon}$ be the set of such packet and energy arrival rate pairs. We will seek to obtain routing and scheduling policies that stabilize the system for any packet and energy arrival rate in $\Lambda_{\delta, \varepsilon}$ for any $\delta, \varepsilon>0$, for large energy storage capacities (Section III). Subsequently, we will quantify the reduction in the network stability region as a function of the energy storage capacity (Section V).

\section{ThroughPUT-OPTIMAL ROUTING AND SCHEDULING}

We first consider a network where all nodes need unit energy per transmission $\left(t_{v}=1\right)$, there is no energy cost for reception $\left(r_{v}=0\right)$, and each node has infinite energy storage capacity $\left(B_{i}=\infty\right)$. Under these idealized assumptions, we describe a routing and scheduling policy that does not require any knowledge of the packet and energy arrival rate vectors, and prove that it stabilizes the network for any packet and energy arrival rate in $\Lambda_{\delta, \varepsilon}$ for any positive $\delta, \varepsilon$. Later in this section, we generalize the policy for scenarios where different nodes need different amounts of energy for transmission and reception, and the reception energy cost may be non-zero. The infinite storage capacity restriction is relaxed in the next section.

\section{A. Joint Routing-Scheduling Policy}

The policy consists of two key steps.

a) Energy Marking: Each node consists of two (virtual) buffers: entry buffer and exit buffer. As the name suggests, the former stores packets of different sessions arriving from the input links of the node and the extraneous packets of each session generated at the node, different sessions in separate queues. Available energy units at the node energy storage are marked for consumption in transmission of packets at the exit buffer. In each slot, consider nodes at which energy buffer has at least one unit of unmarked energy. Calculate the differences in the number of packets in each input queue and its corresponding output queue and select the pair which has the largest difference value of surplus in the input buffer, provided that this value is strictly positive. Among such queues 
(each corresponding to a unique session), one queue is selected (say at random) from which one packet is transferred from the entry buffer to the corresponding queue in the exit buffer. Simultaneously, 1 unit of energy in the energy buffer is marked for this packet. The packet now awaits transmission in one of the output links, and the marked energy unit will be used for transmitting the packet whenever the transmission process occurs. Fig. 1 illustrates this. The marking is of course a logical step, and ensures that the node has enough energy to transmit each packet in its exit buffer, and thus transmissions can be scheduled from this buffer without considering energy availability any further.

b) Packet Transmission: At a scheduled opportunity, a node transmits packets from its exit buffer, according to the following policy. Let $Q_{m u}^{o}(t), Q_{m u}^{i}(t)$ denote the queue lengths of session $m$ at the exit and entry buffers at each node $u$. The weight of each link $(u, v)$ is the largest difference between the queue lengths at the exit buffer of $u$ and the entry buffer of $v$ amongst different sessions, $\max _{m}\left(Q_{m u}^{o}(t)-Q_{m v}^{i}(t)\right)$. The weight of a schedulable set is the sum of the weights of the links in the set. At any slot, the schedulable set with the maximum weight (and minimum size amongst all those with the maximum weight) is found and each link in that set transmits a packet.

Clearly, the energy marking step is localized and as a result no node needs to know of other nodes' energy availabilities. The packet transmission step is in general a high complexity procedure (corresponds to an maximum weight independent set problem in general) due to the global nature of the interference constraints. For certain forms of "local" interference constraints, it can be implemented or approximated in a distributed manner, with low message complexity. See [17] and references therein for a discussion of low message scheduling algorithms with provable approximation guarantees.

The packet transmission step not only selects the sessions and schedules links, but also selects routes for the packets of a session by determining which links they would follow in the immediate next step. The routing depends on both congestion and energy availability in an implicit manner. For example, if a node $u$ is currently generating small amounts of energy, the energy marking step will transfer packets to its exit buffer only at a low rate, and thus the entry buffer for $u$ will build up. As a result, the weights of the incoming links to $u$ will be negative, and hence they will not be scheduled. Thus, $u$ applies back-pressure on upstream nodes due to which packets will not traverse along the paths through $u$. Note that the exit buffer and subsequently the entry buffer at $u$ can build up also because of traffic congestion downstream, which will again lead to lesser use of the paths traversing $u$. Our packet transmission step is very similar to the adaptive backpressure routing policy for congestion avoidance as proposed by Tassiulas et. al. [18]. Our contribution is to show that backpressure can also be used to respond to fluctuations in energy availabilities, through energy marking and maintenance of two buffers at each node instead of one. Accordingly, we name our policy as Energy Back-pressure, and proceed to prove that it attains the maximum possible throughput even when the energy availabilities at nodes randomly vary, increasing due to energy generation and subsequent storage, and decreasing due to packet transmissions.

\section{B. Stability Analysis}

Theorem 1: If $(\vec{\lambda}, \vec{e}) \in \Lambda_{\delta, \varepsilon}$ for some positive $\delta, \varepsilon$, the Energy Back-pressure policy stabilizes the network.

Proof: In this proof, we will consider $\vec{Q}^{\prime}(t)$ as the vector of queue lengths of all sessions at both entry and exit buffers of the nodes. Let also $\vec{A}^{\prime}(t), \vec{D}^{\prime}(t)$ be the vector of arrivals and departures at both entry and exit buffers, considering both arrivals from other nodes and external arrivals. For simplicity of exposition and space limit reasons, we present the proof when the energy arrival process (and the packet arrival process) is temporally independent; energy arrivals (packet arrivals) in different nodes at any slot may be correlated though.

We consider a quadratic lyapunov function: $V(\vec{x})=\sum_{i} x_{i}^{2}$, and investigate its conditional expected drift.

$$
\begin{array}{r}
\mathbf{E}\left[V\left(\vec{Q}^{\prime}(t+1)\right)-V\left(\vec{Q}^{\prime}(t)\right) \mid \vec{Q}^{\prime}(t)\right] \\
=2 \mathbf{E}\left[\left(\vec{Q}^{\prime}(t)\right)^{T}\left(\vec{A}^{\prime}(t)-\vec{D}^{\prime}(t)\right) \mid \vec{Q}^{\prime}(t)\right] \\
+\mathbf{E}\left[\left(\vec{A}^{\prime}(t)-\vec{D}^{\prime}(t)\right)^{T}\left(\vec{A}^{\prime}(t)-\vec{D}^{\prime}(t)\right) \mid \vec{Q}^{\prime}(t)\right] \\
\leq 2 \mathbf{E}\left[\left(\vec{Q}^{\prime}(t)\right)^{T}\left(\vec{A}^{\prime}(t)-\vec{D}^{\prime}(t)\right) \mid \vec{Q}^{\prime}(t)\right] \\
+\mathbf{E}\left[\left(\vec{A}^{\prime}(t)\right)^{T}\left(\vec{A}^{\prime}(t)\right) \mid \vec{Q}^{\prime}(t)\right] \\
+\mathbf{E}\left[\left(\vec{D}^{\prime}(t)\right)^{T}\left(\vec{D}^{\prime}(t)\right) \mid \vec{Q}^{\prime}(t)\right] \\
=2 \mathbf{E}\left[\left(\vec{Q}^{\prime}(t)\right)^{T}\left(\vec{A}^{\prime}(t)-\vec{D}^{\prime}(t)\right) \mid \vec{Q}^{\prime}(t)\right] \\
+\mathbf{E}\left[\sum_{v, m}\left(\sum_{u} D_{u v}^{m}(t)+A_{v}^{m}(t)\right)^{2}+\sum_{u, m}\left(F_{v}^{m}(t)\right)^{2}\right. \\
\left.+\sum_{v, m}\left(F_{v}^{m}(t)\right)^{2}+\sum_{v, m}\left(\sum_{u} D_{v u}^{m}(t)\right)^{2} \mid \vec{Q}^{\prime}(t)\right] \\
\leq 2 \mathbf{E}\left[\left(\vec{Q}^{\prime}(t)\right)^{T}\left(\vec{A}^{\prime}(t)-\vec{D}^{\prime}(t)\right) \mid \vec{Q}^{\prime}(t)\right]+\mu
\end{array}
$$

where $\mu$ is a constant bounded (loosely) by $\left((1+\gamma)^{2}+3\right) N M$, as each of the terms $\left(F_{v}^{m}(t)\right)^{2}$ and $\sum_{u} D_{u v}^{m}(t)$ and $\left(\sum_{u} D_{v u}^{m}(t)\right)^{2}$ are bounded by unity (at any $t$, they are either zero or one), and $A_{v}^{m}(t)$ is bounded by $\gamma$, and the summation is over all $v, m$.

Let $F_{v}^{m}(t)$ be the number of packets that have been transferred from queue of session $m$ in the entry buffer to its corresponding queue in the exit buffer at node $v$ in slot $t$. Following our scheduling policy, $Q_{m v}^{i}(t) \geq Q_{m v}^{o}(t)$ for all $m, v, t$. Recall that $F_{v}^{m}(t)=0$ if $Q_{m v}^{i}(t)=Q_{m v}^{o}(t)$.

$$
\mathbf{E}\left[\left(\vec{Q}^{\prime}(t)\right)^{T}\left(\vec{A}^{\prime}(t)-\vec{D}^{\prime}(t)\right) \mid \vec{Q}^{\prime}(t)\right]
$$




$$
\begin{gathered}
=-\sum_{\substack{(u, v) \in E \\
m \in \mathcal{M}}} \mathbf{E}\left[\left(Q_{m u}^{o}(t)-Q_{m v}^{i}(t)\right) D_{(u, v)}^{m}(t) \mid \vec{Q}^{\prime}(t)\right] \\
+\sum_{\substack{v \in V \\
m \in \mathcal{M}}} \mathbf{E}\left[A_{v}^{m}(t) Q_{m v}^{i}(t) \mid \vec{Q}^{\prime}(t)\right] \\
\sum_{v, m: Q_{m v}^{i}(t)>Q_{m v}^{o}(t)} \mathbf{E}\left[F_{v}^{m}(t)\left(Q_{m v}^{i}(t)-Q_{m v}^{o}(t)\right) \mid \vec{Q}^{\prime}(t)\right] \\
\leq-\max _{\substack{J \in \mathcal{J} \\
m \in \mathcal{M}}} \sum_{\substack{u, v) \in J \\
m \in \mathcal{M}}}\left(Q_{m u}^{o}(t)-Q_{m v}^{i}(t)\right) \\
+\sum_{\substack{v \in V \\
m \in \mathcal{M}}} \lambda_{v}^{m} Q_{m v}^{i}(t)-\sum_{v \in V} e_{v} \alpha_{v}(t)
\end{gathered}
$$

where for each $\alpha_{v}(t):=\max _{m \in \mathcal{M}}\left(Q_{m v}^{i}(t)-Q_{m v}^{o}(t)\right)$. The last inequality follows from energy matching and the packet transmission policy; and observing that irrespective of the size of the energy and packet queues, 1 unit of energy arrives at node $v$ w.p. $e_{v}$, and $F_{v}^{m}(t)=1$ for the queue which is a maximizer of $\left(Q_{m v}^{i}-Q_{m v}^{o}\right)$ and $v$ has at least 1 unit of unmatched energy (including new arrivals). (Note that $v$ may have 1 unit of energy owing to storage even when no energy unit arrives.) We also use the fact that the energy arrival process (and packet arrival process) is temporally independent in this step. Let $J^{O_{v}}=J \cap O_{v}$, and $J^{I_{v}}=J \cap I_{v}$.

$$
\begin{aligned}
& \max _{\substack{J \in \mathcal{J} \\
m \in \mathcal{M}}} \sum_{(u, v) \in J}\left(Q_{m u}^{o}(t)-Q_{m v}^{i}(t)\right) \\
\geq & \sum_{\substack{m \in \mathcal{M} \\
m \in \mathcal{M}}} \omega_{J}^{m} \sum_{(u, v) \in J}\left(Q_{m u}^{o}(t)-Q_{m v}^{i}(t)\right) \\
= & \sum_{\substack{v \in V \\
m \in \mathcal{M}}}\left(\sum_{J \in \mathcal{J}} \omega_{J}^{m}\left|J^{O_{v}}\right| Q_{m v}^{o}(t)-\sum_{J \in \mathcal{J}} \omega_{J}^{m}\left|J^{I_{v}}\right| Q_{m v}^{i}(t)\right)
\end{aligned}
$$

Now, from (8) and the above inequality, we obtain:

$$
\begin{gathered}
\mathbf{E}\left[\left(\vec{Q}^{\prime}(t)\right)^{T}\left(\vec{A}^{\prime}(t)-\vec{D}^{\prime}(t)\right) \mid \vec{Q}^{\prime}(t)\right] \\
\leq-\sum_{\substack{v \in V \\
m \in \mathcal{M}}}\left\{\left(\sum_{J \in \mathcal{J}} \omega_{J}^{m}\left|J^{O_{v}}\right| Q_{m v}^{o}(t)-\sum_{J \in \mathcal{J}} \omega_{J}^{m}\left|J^{I_{v}}\right| Q_{m v}^{i}(t)\right)\right. \\
\left.-\lambda_{v}^{m} Q_{m v}^{i}(t)\right\}-\sum_{v \in V} e_{v} \alpha_{v}(t) \\
=-\sum_{\substack{v \in V \\
m \in \mathcal{M}}}\left\{\left(\sum_{J \in \mathcal{J}} \omega_{J}^{m}\left|J^{O_{v}}\right| Q_{m v}^{i}(t)-\sum_{J \in \mathcal{J}} \omega_{J}^{m}\left|J^{I_{v}}\right| Q_{m v}^{i}(t)\right.\right. \\
\left.-\sum_{v \in V} e_{v} \alpha_{v}(t)+\sum_{\substack{v \in V \\
m \in \mathcal{M}}} \sum_{J \in \mathcal{J}} \omega_{J}^{m}\left|J^{O_{v}}\right|\left(Q_{m v}^{i}(t)-Q_{m v}^{o}(t)\right)\right\}
\end{gathered}
$$

$$
\begin{array}{r}
\leq-\sum_{\substack{v \in V \\
m \in \mathcal{M}}}\left\{\left(\sum_{J \in \mathcal{J}} \omega_{J}^{m}\left|J^{O_{v}}\right| Q_{m v}^{i}(t)-\sum_{\substack{J \in \mathcal{J} \\
m \in \mathcal{M}}} \omega_{J}^{m}\left|J^{I_{v}}\right| Q_{m v}^{i}(t)\right.\right. \\
\left.\left.-\sum_{v \in V}\left(e_{v}-\sum_{\substack{J \in \mathcal{J} \\
m \in \mathcal{M}}} \omega_{J}^{m}\left|J^{O_{v}}\right|\right) \alpha_{v}(t)\right)\right\}
\end{array}
$$

Since $(\vec{\lambda}, \vec{e}) \in \Lambda_{\delta, \varepsilon}, \quad$ from (5), $\quad \sum_{J \in \mathcal{J}} \omega_{J}^{m}\left|J^{O_{v}}\right|-$ $\sum_{J \in \mathcal{J}} \omega_{J}^{m}\left|J^{I_{v}}\right|-\lambda_{v}^{m} \geq \delta$, for all $v, m$; and from (6), $\left(e_{v}-\sum_{\substack{J \in \mathcal{J} \\ m \in \mathcal{M}}} \omega_{J}^{m}\left|J^{O_{v}}\right|\right) \geq \varepsilon$ for all $v$. Also, following our scheduling policy, $Q_{m v}^{i}(t) \geq Q_{m v}^{o}(t), \forall v, k$ and hence $\alpha_{v}(t) \geq 0 \forall v, t$. Therefore, (9) leads to:

$$
\mathbf{E}\left[\left(\vec{Q}^{\prime}(t)\right)^{T}\left(\vec{A}^{\prime}(t)-\vec{D}^{\prime}(t)\right) \mid \vec{Q}^{\prime}(t)\right] \leq-\delta \sum_{v, m} Q_{m v}^{i}(t)
$$

Thus, from (7), (10),

$$
\mathbf{E}\left[V\left(\vec{Q}^{\prime}(t+1)\right)-V\left(\vec{Q}^{\prime}(t)\right) \mid \vec{Q}^{\prime}(t)\right] \leq \mu-2 \delta \sum_{v, m} Q_{m v}^{i}(t) .
$$

Then, $\mathbf{E}\left[V\left(\vec{Q}^{\prime}(t+1)\right)-V\left(\vec{Q}^{\prime}(t)\right)\right] \leq \mu-2 \delta \sum_{v, m} \mathbf{E}\left[Q_{m v}^{i}(t)\right]$. Taking a telescopic sum from 1 to $T$,

$$
\mathbf{E}\left[V\left(\vec{Q}^{\prime}(T)\right)-V\left(\vec{Q}^{\prime}(1)\right)\right] \leq \mu T-2 \delta \sum_{t=1}^{T} \sum_{v, m} \mathbf{E}\left[Q_{m v}^{i}(t)\right] .
$$

Thus, $\frac{1}{T} \sum_{t=1}^{T} \sum_{v, m} \mathbf{E}\left[Q_{m v}^{i}(t)\right] \leq \mu / 2 \delta+V(\vec{Q}(1)) / T$. Recalling again that because of our scheduling policy, $Q_{m v}^{i}(t) \geq$ $Q_{m v}^{o}(t) \forall v, k$, we get $\lim _{T \rightarrow \infty} \frac{1}{T} \sum_{t=1}^{T} \sum_{v, m} \mathbf{E}\left[Q_{m v}(t)\right] \leq$ $2 \times \mu / 2 \delta=\mu / \delta$.

\section{Delay Bounds}

We bound the order of the delay achieved by our scheduling policy. However, we make an additional assumption: we assume the routes are preset for each sessions and are such that there is no loop from any source to its destination. ${ }^{1}$

\section{A. Upper Bound}

Theorem 2: For $\lambda \in \Lambda_{\delta, \varepsilon}$ and under the no-loop routes assumption stated above, the expected delay attained by our policy is at most $\frac{((4+2 \gamma) N+3+\gamma)}{4 \delta}$.

Proof: We now use the fact that, as we proved in theorem 1 , our scheduling policy stabilizes the network, and

\footnotetext{
${ }^{1}$ In the absence of this assumption, backpressure can lead to loops in the low-load multihop scenarios, and despite the boundedness of the summation of the queue lengths, no delay-bound is easy to achieve.
} 
we investigate the drift anew. Now,

$$
\begin{array}{r}
\mathbf{E}\left[V\left(\vec{Q}^{\prime}(t+1)\right)-V\left(\vec{Q}^{\prime}(t)\right)\right] \\
=2 \mathbf{E}\left[\left(\vec{Q}^{\prime}(t)\right)^{T}\left(\vec{A}^{\prime}(t)-\vec{D}^{\prime}(t)\right)\right] \\
+\mathbf{E}\left[\left(\vec{A}^{\prime}(t)-\vec{D}^{\prime}(t)\right)^{T}\left(\vec{A}^{\prime}(t)-\vec{D}^{\prime}(t)\right)\right] \\
=2 \mathbf{E}\left[\left(\vec{Q}^{\prime}(t)\right)^{T}\left(\vec{A}^{\prime}(t)-\vec{D}^{\prime}(t)\right)\right] \\
+\mathbf{E}\left[\sum_{v, m}\left(\sum_{u} D_{u v}^{m}(t)+A_{v}^{m}(t)-F_{v}^{m}(t)\right)^{2}\right. \\
\left.+\sum_{v, m}\left(F_{v}^{m}(t)-\sum_{u} D_{v u}^{m}(t)\right)^{2}\right]
\end{array}
$$

We can bound the first term using (10):

$$
2 \mathbf{E}\left[\left(\vec{Q}^{\prime}(t)\right)^{T}\left(\vec{A}^{\prime}(t)-\vec{D}^{\prime}(t)\right)\right] \leq-2 \delta \mathbf{E} \sum_{v, m} Q_{m v}^{i}(t)
$$

Now, let us investigate the other term:

$$
\begin{aligned}
& \mathbf{E}\left[\sum_{v, m}\left(\sum_{u} D_{u v}^{m}(t)+A_{v}^{m}(t)-F_{v}^{m}(t)\right)^{2}\right. \\
& \left.+\sum_{v, m}\left(F_{v}^{m}(t)-\sum_{u} D_{v u}^{m}(t)\right)^{2}\right] \\
& \leq \mathbf{E}\left[\sum _ { v , m } \left\{\left(\sum_{u} D_{u v}^{m}(t)\right)^{2}+\left(A_{v}^{m}(t)\right)^{2}\right.\right. \\
& \quad+2\left(\sum_{u} D_{u v}^{m}(t)\right) A_{v}^{m}(t) \\
& \left.\quad+\left(F_{v}^{m}(t)\right)^{2}+\left(F_{v}^{m}(t)\right)^{2}+\left(\sum_{u} D_{v u}^{m}(t)\right)^{2}\right\}
\end{aligned}
$$

Note that in our scheduling policy, for all $t, m, v, F_{v}^{m}(t) \in$ $\{0,1\}$. Also, $\sum_{u} D_{u, v}^{m}(t) \in\{0,1\}$. Likewise, $\sum_{u} D_{v, u}^{m}(t) \in$ $\{0,1\}$. Thus, the R.H.S in the above is:

$$
\begin{aligned}
= & \mathbf{E}\left[\sum _ { v , m } \left\{\left(\sum_{u} D_{u v}^{m}(t)\right)+\left(A_{v}^{m}(t)\right)^{2}+2\left(\sum_{u} D_{u v}^{m}(t)\right) A_{v}^{m}(t)\right.\right. \\
& \left.+F_{v}^{m}(t)+F_{v}^{m}(t)+\left(\sum_{u} D_{v u}^{m}(t)\right)\right\} \\
\leq & \sum_{v, m}\left((4+2 \gamma) \mathbf{E}\left(\sum_{u} D_{u v}^{m}(t)\right)+3 \mathbf{E} A_{v}^{m}(t)+\mathbf{E}\left(A_{v}^{m}(t)\right)^{2}\right) \\
\leq & \sum_{v, m}\left((4+2 \gamma)\left(\sum_{w} \lambda_{w}^{m}\right)+3 \lambda_{v}^{m}+\gamma \lambda_{v}^{m}\right) \\
\leq & ((4+2 \gamma) N+3+\gamma) \sum_{v, m} \lambda_{v}^{m}
\end{aligned}
$$

where in the last two steps, we have used the fact that $\mathbf{E} F_{v}^{m}(t)=\mathbf{E}\left(A_{v}^{m}(t)+\sum_{u} D_{u v}^{m}(t)\right)$, and also $\mathbf{E} \sum_{u} D_{v u}^{m}(t)=$
$\mathbf{E}\left(A_{v}^{m}(t)+\sum_{u} D_{u v}^{m}(t)\right)$. Finally, noting that following the assumption of fixed routes with no loops, we have:

$$
\mathbf{E}\left(\sum_{u} D_{u v}^{m}(t)\right) \leq \sum_{w} \lambda_{w}^{m}
$$

Note also that $E\left(A_{v}^{m}(t)\right)^{2} \leq \gamma \mathbf{E} A_{v}^{m}=\gamma \lambda_{v}^{m}$ simply because $\left(A_{v}^{m}(t)\right)^{2} \leq \gamma A_{v}^{m}(t)$.

Note that since the network is stable, we have:

$$
\mathbf{E}\left(V\left(Q^{\prime}(t+1)\right)-V\left(Q^{\prime}(t)\right)\right)=0
$$

Hence, from (12) and (14), we obtain:

$$
\begin{aligned}
0 & \leq-2 \delta \mathbf{E} \sum_{\substack{(u, v) \in J \\
m \in \mathcal{M}}} Q_{m v}^{i}(t)+((4+2 \gamma) N+3+\gamma) \sum_{v, m} \lambda_{v}^{m} \\
& \Rightarrow 2 \delta \mathbf{E} \sum_{\substack{(u, v) \in J \\
m \in \mathcal{M}}} Q_{m v}^{i}(t) \leq((4+2 \gamma) N+3+\gamma) \sum_{v, m} \lambda_{v}^{m} \\
& \Rightarrow 4 \delta \mathbf{E} \sum_{\substack{(u, v) \in J \\
m \in \mathcal{M}}} Q_{m v}(t) \leq((4+2 \gamma) N+3+\gamma) \sum_{v, m} \lambda_{v}^{m}
\end{aligned}
$$

Hence

$$
\mathbf{E} \sum_{\substack{(u, v) \in J \\ m \in \mathcal{M}}} Q_{m v}(t) \leq \frac{((4+2 \gamma) N+3+\gamma) \sum_{v, m} \lambda_{v}^{m}}{4 \delta} .
$$

Since the expected delay (by Little's law) is $\left(\mathbf{E} \sum_{v, m} Q_{m v}(t)\right) /\left(\sum_{v, m} \lambda_{v}^{m}\right)$, the result follows.

\section{B. Generalizations}

When nodes consume different amounts of energy for transmissions, i.e., $t_{v}$ differs across nodes, a necessary condition for transferring a packet to the exit buffer at $v$ is that $v$ has at least $t_{v}$ amount of unmarked energy and such a transfer leads to marking of $t_{v}$ energy units at $v$. With this small change in policy, Theorem 1 holds and can be proved similarly.

When reception energy cost $r_{v}$ is non-negligible, the packet buffering policy has to be altered slightly, as we explain next. In this case, a node not only can not transmit a packet if it has no residual energy, it can not receive a packet either. The energy-marking step in energy back-pressure policy need to be modified as follows. Each node $u$ maintains one entry buffer as before, but one intermediate buffer and $\left|O_{u}\right|$ exit buffers, one for each of its outgoing links. A packet is transferred from the entry buffer to the intermediate buffer if (i) $u$ has at least $t_{v}$ units of unmarked energy (including new energy arrivals), and (ii) the intermediate buffer at $u$ has fewer packets than the entry-buffer; an energy unit is marked for usage at $u$ if a packet is transferred. Thus, the intermediate buffer plays the role of exit buffer in the earlier version. A packet is transferred from the intermediate buffer to an exit buffer only if (a) the endnode of the corresponding outgoing link has at least $r_{v}$ units of unmarked energy, and (b) the exit buffer has fewer packets than the intermediate buffer. Thus, a packet is transferred to an 
exit buffer only if the corresponding end-node has energy to receive it, and then an energy unit is marked at this end-node. Note that this step requires a node to communicate with its next hop (unlike the case where only transmission energy cost is considered), but such message exchange is still "local". At most 1 packet is transferred to the exit buffers at $u$ in any slot, and the one that has the least number of packets among all exit buffers that satisfy both (a) and (b) is selected. The scheduling step does not change, except that the weight of each link $(u, v)$ is now the difference between the queue lengths at the exit buffer of $u$ corresponding to $v$ ( $u$ will in general have several exit buffers) and the entry buffer of $v$. Theorem 1 continues to hold - the proof, which is similar to the one presented earlier, is omitted for brevity.

\section{NEAR-OPTIMAL THROUGHPUT IN PRESENCE OF FINITE ENERGY STORAGE}

We now consider a network where nodes can store only finite amount of energy. In particular, each node $v$ can store $B$ units of energy, and new energy that is generated while the storage is full will be wasted if not utilized instantaneously. For simplicity of exposition, we assume that there is only one session in the network. Specifically, all nodes inted to deliver their generated packets eventually to a single sink node. We prove that any feasible arrival and energy rate pair in $\Lambda_{\delta}$ is stabilized as long as $B \geq 4 / \delta$, and describe a routing and scheduling policy that attains this goal without requiring any knowledge of the packet and energy arrival rate vectors. Note that $\delta$ represents the arrival slack, the "distance" of the arrival rate vectors from the boundary of the stability region - thus, storage requirements increase with arrival slack decreases. The trend is of course anticipated, but our results quantify the relation.

\section{A. Policy Description}

To motivate the development and significance of our result in this context, let us start out examining the energy backpressure policy that uses infinite energy storage. Note that whenever a packet is transferred to an exit buffer, an energy unit is marked for its transmission and must be stored until the packet is transmitted. We assumed that these buffers could hold any number of packets, and thus the energy storage must be infinite. Arguing this way, the size of these buffers could be related to the amount of energy storage energy back-pressure can use in a finite energy storage scenario. Thus, some of the recent work that quantifies throughput loss in presence of finite packet buffers, as [22], [23] can be leveraged to address the case of finite energy storage. These papers however provide throughput optimal policies (in networks where nodes have unlimited energy) when packet buffers increase linearly with increase in the number of nodes [23], or number of hops in the network [22] (in addition to increasing with decrease in arrival slack). Both of these quantities can be very large for multi-hop wireless networks, and energy storage per node is limited (and independent of the network size in general), and thus these policies do not apply. We now describe the
Finite-storage Energy Back-pressure policy that we develop for maximizing the throughput while using energy storage amounts that depend only on the arrival slack (and not on network size parameters). The design of this policy exploits the fact that packet buffer storage is unlimited in our case, only energy storage is limited. Again, for simplicity we consider $t_{v}=1, r_{v}=0, l_{v}=B$ and our results can be generalized as in the previous section when these assumptions are relaxed.

We describe how finite storage energy back pressure policy differs from its infinite storage version. The energy marking storage step is the same except that a packet at the entry buffer of node $v$ is transferred to the exit buffer if $v$ has at least one unit of unmarked energy and the exit buffer has fewer than $B$ packets (note that a packet may be transferred even if the entry buffer has fewer packets than the exit buffer). The packet transmission step is the same except that the weight of link $(u, v)$ at time $t$ is $Q_{u}^{o}(t) Q_{u}^{i}(t)-B Q_{v}^{i}(t)$. Note that $Q_{u}^{o}(t) \leq B$, and thus the queue length at the entry buffer of $u$ is weighed less than that for the exit buffer in calculating this weight. We next prove that this policy stabilizes the network for any packet and energy arrival rate vectors in $\Lambda_{4 / B}$ (i.e., as long as he arrival slack is $4 / B$ or higher).

\section{B. Stability Analysis}

Theorem 3: If $(\vec{\lambda}, \vec{e}) \in \Lambda_{\delta}$ and $B \geq 4 / \delta$, the Finite-storage Energy Back-pressure policy stabilizes the network.

Proof: Let $\vec{Q}^{\prime}(t), F_{v}(t), J^{O_{v}}, J^{I_{v}}$ be as defined in the proof of Theorem 1. Again, we present the proof when the energy and packet arrival processes are temporally independent, although it can be easily generalized to stationary ergodic processes. We investigate the conditional expected drift of the lyapunov function: $V\left(\vec{Q}^{\prime}\right)=\sum_{u}\left(Q_{u}^{i}\right)^{2}+(1 / B) \sum_{u}\left(Q_{u}^{o}\right)^{2} Q_{u}^{i}$.

$$
\mathbf{E}\left[V\left(\vec{Q}^{\prime}(t+1)\right)-V\left(\vec{Q}^{\prime}(t)\right) \mid \vec{Q}^{\prime}(t)\right]=\Delta R_{1}+\Delta R_{2},
$$

$$
\begin{aligned}
& \text { where } \Delta R_{1}=\mathbf{E}\left[\sum_{u}\left(Q_{u}^{i}(t+1)\right)^{2}-\left(Q_{u}^{i}(t)\right)^{2} \mid \vec{Q}^{\prime}(t)\right] \\
& \qquad \begin{aligned}
\text { and } \Delta R_{2}= & (1 / B) \mathbf{E}\left[\sum_{u}\left(Q_{u}^{o}(t+1)\right)^{2} Q_{u}^{i}(t+1)\right. \\
& \left.-\left(Q_{u}^{o}(t)\right)^{2} Q_{u}^{i}(t) \mid \vec{Q}^{\prime}(t)\right] .
\end{aligned}
\end{aligned}
$$

Now, $\Delta R_{1}$ is at most

$$
\mathbf{E}\left[\sum_{u} Q_{u}^{i}(t)\left(A_{u}(t)+\sum_{v \in I_{u}} D_{v}(t)-F_{u}(t)\right) \mid \vec{Q}^{\prime}(t)\right]+\mu_{1}
$$


here $\mu_{1}$ is a constant that depends on $M, N, L, \gamma$.

$$
\begin{aligned}
& \sum_{u}\left(Q_{u}^{o}(t+1)\right)^{2} Q_{u}^{i}(t+1) \\
= & \sum_{u}\left(Q_{u}^{o}(t)+F_{u}(t)-\sum_{v \in O_{u}} D_{v}(t)\right)^{2} \\
& \times\left[Q_{u}^{i}(t)+A_{u}(t)+\sum_{v \in I_{u}} D_{v}(t)-F_{u}(t)\right] \\
\leq \quad & \left(Q_{u}^{o}(t)\right)^{2} Q_{u}^{i}(t)+\left(F_{u}(t)-\sum_{v \in O_{u}} D_{v}(t)\right)^{2} Q_{u}^{i}(t) \\
& +2 Q_{u}^{i}(t) Q_{u}^{o}(t)\left(F_{u}(t)-\sum_{v \in O_{u}} D_{u}(t)\right)+\mu_{2},
\end{aligned}
$$

where $\mu_{2}$ is a constant that depends on $M, N, L, \gamma, B$ (we use the fact that $\left.Q_{u}^{o}(t) \leq B, F_{u}(t) \leq 1, \sum_{v \in O_{u}} D_{v}(t) \leq 1\right)$. Again, using the same, $\Delta R_{2}$ can be upper-bounded by,

$\frac{\mathbf{E}\left[4 Q_{u}^{i}(t)+2 Q_{u}^{i}(t) Q_{u}^{o}(t)\left(F_{u}(t)-\sum_{v \in O_{u}} D_{v}(t)\right) \mid \vec{Q}^{\prime}(t)\right]+\mu_{2}}{B}$.

From (16) and (17),

$$
\begin{gathered}
\Delta R_{1}+\Delta R_{2} \leq(4 / B) \sum_{u} Q_{u}^{i}(t)+\mu_{1}+\left(\mu_{2} / B\right) \\
+2 \mathbf{E}\left[\sum_{u} Q_{u}^{i}(t)\left(A_{u}(t)+\sum_{v \in I_{u}} D_{v}(t)-F_{u}(t)\right)\right. \\
\left.+(2 / B)\left(Q_{u}^{i}(t) Q_{u}^{o}(t)\left(F_{u}(t)-\sum_{v \in O_{u}} D_{v}(t)\right)\right) \mid \vec{Q}^{\prime}(t)\right] .
\end{gathered}
$$

We next upper bound the last term of the R.H.S above, denoted by $S$, henceforth.

$$
\begin{aligned}
& S= 2 \sum_{u} Q_{u}^{i}(t) \lambda_{u} \\
&-(2 / B) \sum_{u} \mathbf{E}\left[F_{u}(t) \mid \vec{Q}^{\prime}(t)\right] Q_{u}^{i}(t)\left(B-Q_{u}^{o}(t)\right) \\
&-(2 / B) \sum_{(u, v) \in E} \mathbf{E}\left[D_{(u, v)}(t) \mid \vec{Q}^{\prime}(t)\right]\left(Q_{u}^{i}(t) Q_{u}^{o}(t)-B Q_{v}^{i}(t)\right) \\
& \leq \quad 2 \sum_{u} Q_{u}^{i}(t) \lambda_{u}-(2 / B) \sum_{u} e_{u} Q_{u}^{i}(t)\left(B-Q_{u}^{o}(t)\right) \\
& \quad-(2 / B) \max _{J \in \mathcal{J}} \sum_{(u, v) \in J}\left(Q_{u}^{i}(t) Q_{u}^{o}(t)-B Q_{v}^{i}(t)\right)
\end{aligned}
$$

We use the fact that the packet arrival process is temporally independent in the first step. The last inequality follows from the packet transmission policy and the temporal independence of the energy arrival process. We obtain it observing that (i) $Q_{u}^{o}(t) \leq B$, and (ii) irrespective of the size of the energy and packet queues 1 unit of energy arrives at node $i$ w.p. $e_{u}$, and (iii) $F_{u}(t)=1$ whenever $Q_{u}^{o}(t)<B$ and $v$ has at least 1 unit of unmatched energy (including new arrivals). Also,

$$
\begin{aligned}
& \max _{J \in \mathcal{J}} \sum_{(u, v) \in J}\left(Q_{u}^{i}(t) Q_{u}^{o}(t)-B Q_{v}^{i}(t)\right) \\
\geq & \sum_{J \in \mathcal{J}} \omega_{J} \sum_{(u, v) \in J}\left(Q_{u}^{i}(t) Q_{u}^{o}(t)-B Q_{v}^{i}(t)\right) \\
\geq & \sum_{u} Q_{u}^{i}(t)\left(Q_{u}^{o}(t) \sum_{J \in \mathcal{J}} \omega_{J}\left|J^{O_{u}}\right|-B \sum_{J \in \mathcal{J}} \omega_{J}\left|J^{I_{u}}\right|\right) . \\
S \leq & 2\left[\sum _ { u } Q _ { u } ^ { i } ( t ) \left(\lambda_{u}+\sum_{J \in \mathcal{J}} \omega_{J}\left|J^{I_{u}}\right|\right.\right. \\
\leq & \left.\left.-\left(Q_{u}^{o}(t) / B\right) \sum_{J \in \mathcal{J}} \omega_{J}\left|J^{O_{u}}\right|-e_{u}\left(1-Q_{u}^{o}(t) / B\right)\right)\right] \\
\leq & \sum_{u} Q_{u}^{i}(t)\left(\sum_{J \in \mathcal{J}} \omega_{J}\left|J^{O_{u}}\right|-\delta-\left(Q_{u}^{o}(t) / B\right) \sum_{J \in \mathcal{J}} \omega_{J}\left|J^{O_{u}}\right|\right. \\
- & \left.\left.\sum_{J \in \mathcal{J}} \omega_{J}\left|J^{O_{u}}\right|\left(1-Q_{u}^{o}(t) / B\right)\right)\right] \quad(\text { from (5) and }(6)) . \\
= & -2 \delta \sum_{u} Q_{u}^{i}(t) .
\end{aligned}
$$

Thus, from (18),

$$
\Delta R_{1}+\Delta R_{2} \leq((4 / B)-2 \delta) \sum_{u} Q_{u}^{i}(t)+\mu_{1}+\left(\mu_{2} / B\right) .
$$

Now, from (15) and observing that $B \geq 4 / \delta$,

$\mathbf{E}\left[V\left(\vec{Q}^{\prime}(t+1)\right)-V\left(\vec{Q}^{\prime}(t)\right) \mid \vec{Q}^{\prime}(t)\right] \leq-\delta \sum_{u} Q_{u}^{i}(t)+\mu_{1}+\left(\mu_{2} / B\right)$.

Now, following the steps after (11) in the proof of Theorem 1,

$$
\lim _{T \rightarrow \infty} \frac{\sum_{t=1}^{T} \sum_{u} \mathbf{E}\left[Q_{u}^{i}(t)\right]}{T} \leq(1 / \delta)\left(\mu_{1}+\mu_{2} / B\right) .
$$

Since $Q_{u}(t)=Q_{u}^{i}(t)+Q_{u}^{o}(t)$ and $Q_{u}^{o}(t) \leq B$,

$$
\lim _{T \rightarrow \infty} \frac{\sum_{t=1}^{T} \sum_{u} \mathbf{E}\left[Q_{u}(t)\right]}{T} \leq B+(1 / \delta)\left(\mu_{1}+\mu_{2} / B\right) .
$$

The result in Theorem 3 can be interpreted in two equivalent ways. It states a minimum energy storage requirement at each node to attain maximum throughput for any given subset of the network stability region. Alternatively, for a given finite energy storage capacity, it quantifies the amount of loss in the maximum throughput due to the limitation on energy storage. This can be useful in provisioning the energy storage capacities in a renewable energy network, or calculating what data rates can be supported in such networks. Also, note that Theorem 3 and its proof assumes at any node can transmit at most one packet transmission in a slot; if a node can transmit up to $\kappa$ packets in a slot, the minimum energy storage requirement for maximum throughput will scale as $O\left(\kappa^{2} / \delta\right)$. The scaling of energy storage limit inversely with $\delta$ is possibly unavoidable, particularly for back-pressure based policies. However, the arrival slack is typically large since the minimum packet delay achievable would also depend inversely on $\delta$; so the energy storage requirement is small for all low-delay traffic loads. 


\section{REFERENCES}

[1] Meraki solar. http://meraki.com/products_services/access_points/ solar_overview/.

[2] Proxim Wireless, "The solar-power alternative in broadband wireless networks," Whitepaper at http://downloads.lightreading.com/wplib/ proxim/Proxim_SolarPowerWP_042407.pdf, 2007.

[3] Aerovironment architectural wind microturbines. http://www. goodcleantech.com/2008/06/aerovironmentarchitecturalwi.php.

[4] R. A. Kellog and J. K. Brotz, "Mechanical vibration to electrical energy converter," US Patent 7498681, March 2009.

[5] V. Raghunathan, A. Kansal, J. Hsu, J. Friedman, and M. Srivastava, "Design considerations for solar energy harvesting wireless embedded systems," in Proc. IPSN, Apr. 2005, pp. 457462.

[6] M. Rahimi, H. Shah, G.S. Sukhatme, J. Heidemann, and D. Estrin, "Studying the feasibility of energy harvesting in a mobile sensor network," in IEEE International Conference on Robotics and Automation, 2003.

[7] X. Jiang, J. Polastre, and D. Culler, "Perpetual environmentally powered sensor networks," in Proc. IPSN, Apr. 2005, pp. 463468.

[8] T. Voigt, H. Ritter, andJ. Schiller, "Utilizing Solar Power inWireless Sensor Networks," in Proc. LCN, 2003, p. 416.

[9] B. C. Norman, "Power options for wireless sensor networks," IEEE Aerospace and Electronic Systems Magazine, vol. 22, no. 4, pp. 1417, Apr. 2007.

[10] A. Kansal, D. Potter, and M. Srivastava, "Performance aware tasking for environmentally powered sensor networks," ACM SIGMETRICS Performance Evaluation Review, vol. 32, no. 1, pp. 223234, Jun. 2004.

[11] K. Kar, A. Krishnamurthy, and N. Jaggi, "Dynamic node activation in networks of rechargeable sensors," IEEE/ACM Transactions on Networking, vol. 14, no. 1, pp. 1526, Feb. 2006.

[12] C. M. Vigorito, D. Ganesan, and A. G. Barto, "Adaptive control of duty cycling in energy-harvesting wireless sensor networks," in IEEE SECON, 2007.

[13] T. Banerjee and A. A. Kherani, "Sensor node activation policies using partial or no information," in WiOpt, Limassol, Apr. 2007, pp. 17.

[14] M. Gatzianas, L. Georgiadis, and L. Tassiulas, "Asymptotically optimal policies for wireless networks with rechargeable batteries," in Proc. Wireless Communications and Mobile Computing Conference (IKCMC), Aug. 2008, pp. 3338.

[15] L. Lin, N. Shroff, and R. Srikant, "Asymptotically optimal power-aware routing for multihop wireless networks with renewable energy sources," in Proc. IEEE INFOCOM, 2005, pp. 12621272.

[16] R-S. Liu, P. Sinha, and E. Koksal "Joint Energy Management and Resource Allocation in Rechargeable Sensor Networks", Proc. of IEEE INFOCOM, San Diego, March 2010.

[17] S. Sarkar and S. Ray, "Arbitrary Throughput Versus Complexity Tradeoffs in Wireless Networks using Graph Partitioning," IEEE Transactions on Automatic Control, Vol. 53, No. 10, November, 2008, pp. 2307-2323.

[18] L. Tassiulas and A. Ephremides, "Stability properties of constrained queueing systems and scheduling policies for maximum throughput in multihop radio networks", IEEE Transactions on Automatic Control, 37(12), December 1992.

[19] Tassiulas, A. Ephremides, "Dynamic server allocation to parallel queues with randomly varying connectivity," IEEE Transactions on Information Theory, Vol. 39, No. 2, pp. 466-478, March 1993.

[20] M. Neely, "Energy optimal control for time varying wireless networks," IEEE transactions on Information Theory, vol. 52, no. 7, pp. 2915-2934, July 2006.

[21] M. H. R Khouzani and S. Sarkar, "Economy of Spectrum Access in Time Varying Multi-Channel Networks," To appear in IEEE Transactions on Mobile Computing, 2010; DOI: 10.1109/TMC.2010.90.

[22] P. Giaccone, E. Leonardi and D. Shah, "Throughput Region of FiniteBuffered Networks," IEEE Transactions on Parallel and Distributed Systems, vol. 18, no. 2, pp. 251-263, Feb. 2007.

[23] L. B. Le, E. Modiano and N. B. Shroff, "Optimal Control of Wireless Networks with Finite Buffers," Proc. of IEEE INFOCOM, San Diego, March 2010 